\title{
Effects of Realistic Surface Topography on Seismic Ground Motion in the Yangminshan Region of Taiwan Based Upon the Spectral-Element Method and LiDAR DTM
}

\author{
by Shiann-Jong Lee, Yu-Chang Chan, Dimitri Komatitsch," \\ Bor-Shouh Huang, and Jeroen Tromp ${ }^{\dagger}$
}

\begin{abstract}
We combine light detection and ranging (LiDAR) digital terrain model (DTM) data and an improved mesh implementation to investigate the effects of highresolution surface topography on seismic ground motion based upon the spectralelement method. In general, topography increases the amplitude of shaking at mountain tops and ridges, whereas valleys usually have reduced ground motion, as has been observed in both records from past earthquakes and numerical simulations. However, the effects of realistic topography on ground motion have not often been clearly characterized in numerical simulations, especially the seismic response of the true ground surface. Here, we use LiDAR DTM data, which provide two-meter resolution at the free surface, and a spectral-element method to simulate three-dimensional (3D) seismic-wave propagation in the Yangminshan region in Taiwan, incorporating the effects of realistic topography. A smoothed topographic map is employed beneath the model surface in order to decrease mesh distortions due to steep ground surfaces. Numerical simulations show that seismic shaking in mountainous areas is strongly affected by topography and source frequency content. The amplification of ground motion mainly occurs at the tops of hills and ridges whilst the valleys and flat-topped hills experience lower levels of ground shaking. Interaction between small-scale topographic features and high-frequency surface waves can produce unusually strong shaking. We demonstrate that topographic variations can change peak ground acceleration (PGA) values by $\pm 50 \%$ in mountainous areas, and the relative change in PGA between a valley and a ridge can be as high as a factor of 2 compared to a flat surface response. This suggests that high-resolution, realistic topographic features should be taken into account in seismic hazard analysis, especially for densely populated mountainous areas.
\end{abstract}

Introduction

It has long been known that surface topography can significantly affect earthquake ground motion. Several numerical studies have indicated that structures located at the tops of hills, ridges, and canyons suffer more intense damage than structures situated at the base of hills (Boore et al., 1972, 1981; Geli et al., 1988; Scott et al., 1997; Ma et al., 2007). This phenomenon has also been observed in past earthquakes, such as the 1986 Hualien earthquake (Chiu et al.,

\footnotetext{
"Also at Institut universitaire de France, 103 boulevard Saint-Michel, 75005 Paris, France.

Now at Department of Geosciences, Princeton University, Princeton, New Jersey.
}

1992), the 1989 Loma Prieta earthquake (Hartzell et al., 1994), and the 1999 Chi-Chi earthquake (Huang, 2000).

In this study, we focus on surface topography effects in the Shamao Mountain area (Fig. 1), which is located in Yangmingshan National Park in northern Taiwan. The park is characterized by a series of volcanoes, named the Datun volcano group. This region is a major outdoor recreation area for the citizens of Taipei, and it is also a densely populated suburb of metropolitan Taipei. Shamao Mountain, a parasitical volcano of the Datun volcano group, is a conical volcano shaped by lava deposits and aggregate rocks spewed during eruptions. The steep peaks of Shamao Mountain are the most striking features of this conical volcano. It is a round volcanic dome looking somewhat like a black gauze cap. The area 


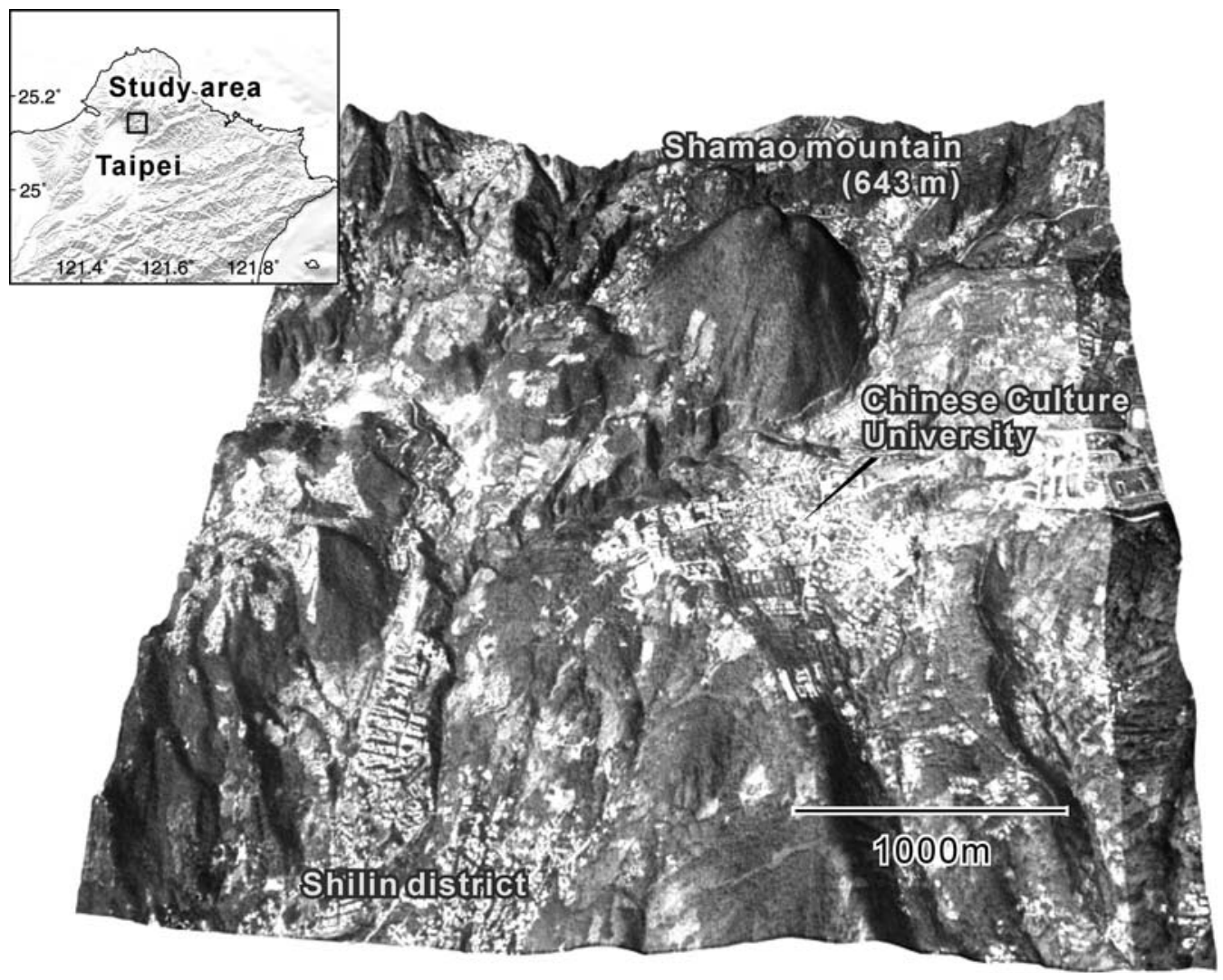

Figure 1. LiDAR 3D digital map of northern Taiwan. A $1 / 5000$ aerial picture is mapped on the $1 \mathrm{~m} \times 1 \mathrm{~m}$ LiDAR digital terrain model. The steep peak of Shamao Mountain, the high population density at the Chinese Culture University, and the Shilin district are the most striking features in the region.

is frequented by hikers, contains a university (the Chinese Culture University), and is located next to the Shilin district, a densely populated outer suburb of Taipei. Lin, Konstantinou, $\mathrm{Pu}$, et al. (2005) and Lin, Konstantinou, Liang, et al. (2005) presented preliminary results from seismic monitoring of the Datun volcano region that indicates seismic activity beneath the Datun volcanic group characteristic of an active volcanic region. Their results were based upon comparisons with seismic activity studies conducted in known active volcanic regions in other parts of the world. The possibility of volcanic activity and the presence of steep surface topography make this populated region particularly vulnerable to volcanic and seismic hazards.

Lee, Chen, Liu, et al. (2008) used the spectral-element method (SEM) to simulate seismic-wave propagation in the Taipei basin to investigate interactions between the basin and surrounding mountains. The surface resolution in their study was about $30 \mathrm{~m}$, which is small enough to resolve $40 \mathrm{~m}$ digital elevation model (DEM) data in Taiwan. The maximum simulated seismic-wave frequency in the study was approximately $1 \mathrm{~Hz}$. To accommodate high-resolution surface topography data from the light detection and ranging (LiDAR) digital terrain model (DTM), which has a resolution close to $2 \mathrm{~m}$, we use the SEM to simulate seismic-wave propagation for frequencies up to $10 \mathrm{~Hz}$ in the Shamao Mountain area.
The SEM is a numerical technique introduced more than 20 years ago to address problems in computational fluid dynamics (Patera, 1984). It is based upon a weak formulation of the equations of motion and naturally incorporates topography. Komatitsch and Vilotte (1998) and Komatitsch and Tromp (1999) provide a detailed introduction to the SEM for 3D seismic-wave propagation. The method has been subsequently applied in many areas of seismology (see, e.g., Komatitsch et al. [2002], [2004] and Chaljub et al. [2003]). The biggest challenge for the successful application of the SEM in the case of complex geological structures lies in the design of the mesh (Komatitsch et al., 2005). Lee, Chen, Liu, et al. (2008) presented a new mesh implementation to improve mesh quality and related numerical stability. Based upon their implementation, realistic topography and complex subsurface structures can be efficiently incorporated within the SEM mesh. In this study, we use the same approach to incorporate high-resolution LiDAR DTM data at the top of the SEM mesh model.

A small earthquake $\left(M_{\mathrm{L}} 1.8\right)$ occurred on 15 November 2003 beneath the study area at a depth of $4.92 \mathrm{~km}$ and was detected by the local seismic monitoring array (Lin, Konstantinou, $\mathrm{Pu}$, et al., 2005). We adopt its fault plane solution (strike $40^{\circ}$, dip $80^{\circ}$, and rake $-90^{\circ}$ ) from the microearthquake catalog and increase the magnitude to 5.0 to investi- 
gate the seismic response in the presence of realistic surface topography in the case of a possibly larger future event.

\section{LiDAR DTM Data and the SEM Mesh}

Realistic Surface Topography from

\section{LiDAR DTM Data}

The airborne LiDAR (Wehr and Lohr, 1999) system consists of an integration of three technologies, namely, the inertial navigation system (INS), LASER, and the global positioning system (GPS). The system is mounted on an aircraft. During flight, the LiDAR sensor pulses a narrow highfrequency laser beam towards Earth; some of the light is reflected and scattered back to the LiDAR system. Changes in the properties of the light help in determining the properties of the Earth's surface (Chan et al., 2007). The Taipei metropolitan area LiDAR mapping project is currently underway. It will dramatically improve the resolution of digital elevation modeling for the entire Taipei region. Compared to the $40 \mathrm{~m}$ resolution of the Taiwan DEM, the LiDAR DTM can provide a $1 \mathrm{~m}$ resolution (Fig. 2). To investigate the seismic response with such high-resolution surface topography, we focus on the Shamao Mountain area (about $4000 \times$ $4000 \mathrm{~m}^{2}$ ) where measurement of the LiDAR DTM was completed in 2006 (Chan et al., 2005).

High-Resolution Spectral-Element Meshes Incorporated with LiDAR DTM

An SEM mesh of the Shamao Mountain area is shown in Figure 3. The whole model is about $4247 \mathrm{~m} \times 3941 \mathrm{~m} \times$ $10665 \mathrm{~m}$. Shamao Mountain is located in the northeast part of the study area. We preserve most of the surface characteristics from LiDAR DTM data at the top of the model with a surface resolution of about $2 \mathrm{~m}$. A 3D wave-speed model taken from a travel-time tomography study (Kim et al., 2005) is embedded in the mesh. The resolution of this model is $8 \mathrm{~km}$ in both horizontal directions and $2 \mathrm{~km}$ in depth, which provides the background 3D wave speeds in the study area. Wave speeds for the volume above sea level are assigned by extending the surface values defined at $z=0 \mathrm{~m}$. In order to include realistic topography in the simulations, a thin buffer layer is used to dampen mesh distortion coming from the surface (Lee, Chen, Liu, et al., 2008). We also introduce three doubling mesh layers to accommodate a finer mesh
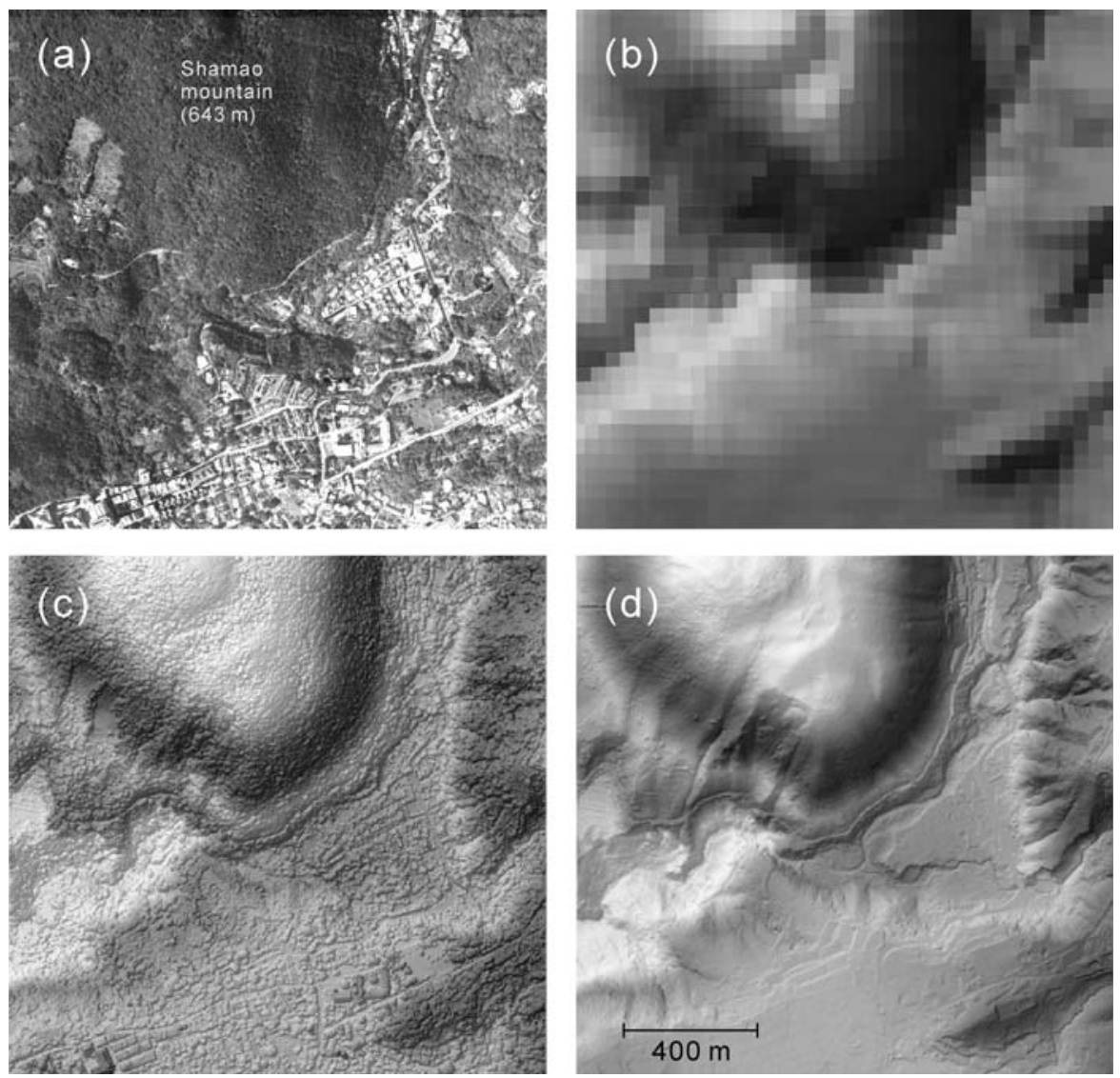

Figure 2. Comparative images of various topographic data sets: (a) 1/5000 aerial topographic map, (b) $40 \mathrm{~m}$ digital elevation model (DEM), (c) $1 \mathrm{~m} \times 1 \mathrm{~m} \mathrm{LiDAR}$ digital surface model (DSM), which includes the presence of vegetation, and (d) $1 \mathrm{~m} \times 1 \mathrm{~m} \mathrm{LiDAR}$ digital terrain model (DTM) without vegetation. 

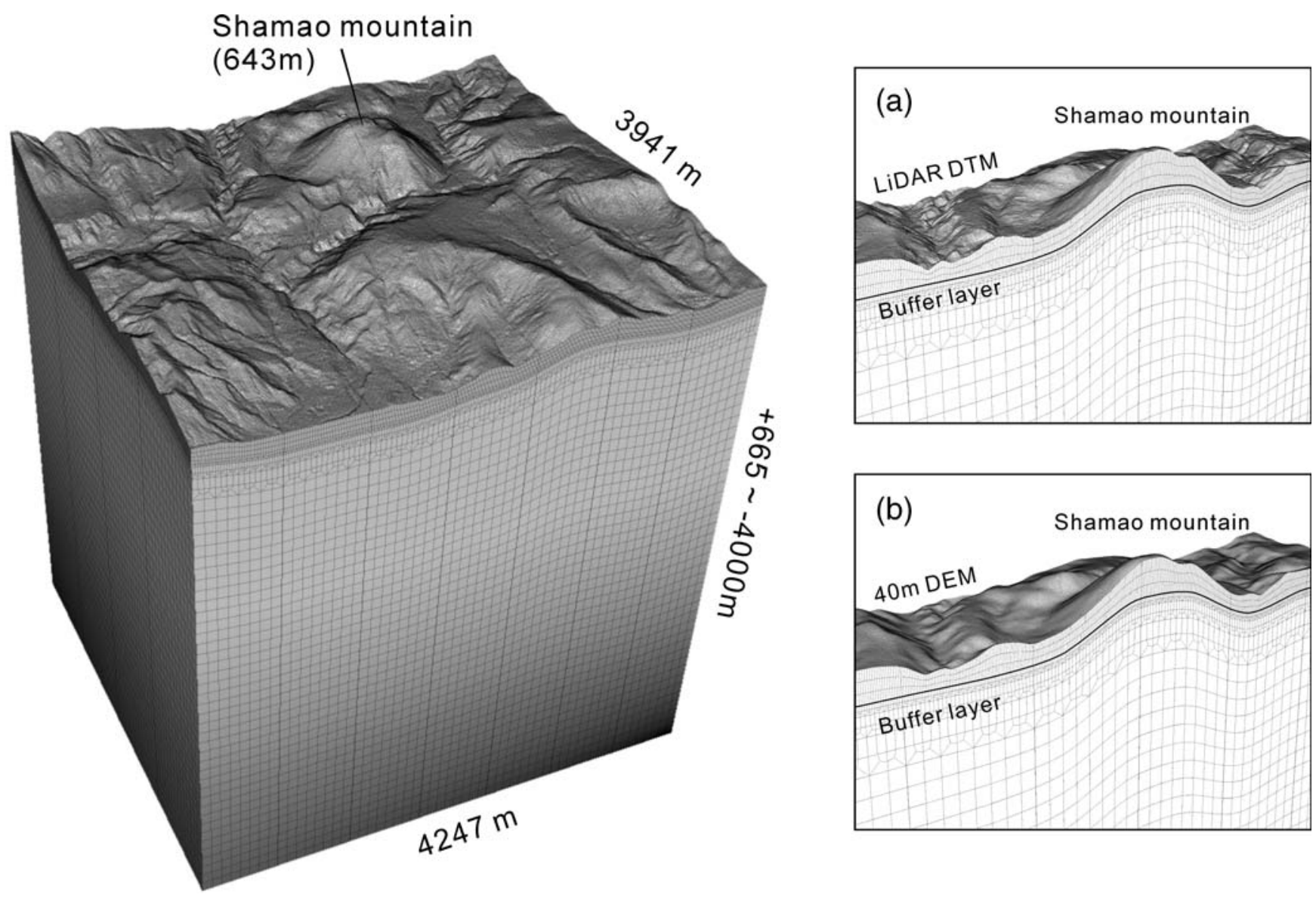

Figure 3. Spectral-element mesh for the study volume. The size of the model is $4247 \mathrm{~m} \times 3941 \mathrm{~m}$ horizontally and $+665 \mathrm{~m}$ to $-10,000 \mathrm{~m}$ vertically ( $+665 \mathrm{~m}$ to $-4000 \mathrm{~m}$ is shown in the figure). Three-dimensional $P$ wave-speed variations are represented by the gray color scale. Shamao Mountain is located close to the middle part of the study area and is characterized by its steep shape. Cross sections of Shamao Mountain generated by two different surface data sets are shown in the right-hand panels: (a) surface topography incorporating $1 \mathrm{~m} \mathrm{LiDAR}$ DTM and (b) surface topography incorporating $40 \mathrm{~m}$ DEM. The average distance between points at the tops of both models is approximately $2 \mathrm{~m}$. Detailed topographic features can be observed in model (a), whereas model (b) shows a smoother ground surface.

near the surface (Komatitsch and Tromp, 1999). Based upon this approach, high-resolution topography obtained from LiDAR DTM data is incorporated without artificial smoothing. Two close-up views that compare the LiDAR DTM and 40 m DEM meshes are shown in Figure 3a,b. Shamao Mountain, along with other surface features, including details of mountain ridges and valleys, can be clearly identified in the LiDAR DTM mesh (Fig. 3a) but not in the $40 \mathrm{~m}$ DEM mesh (Fig. 3b). Becaue of lack of information regarding seismic attenuation, we use a shear quality factor of $Q_{\mu}=500$ in the study area, which is the value used in the bedrock by Lee, Chen, Liu, et al. (2008).

\section{Simulation Results}

We use the SPECFEM3D software package developed by Komatitsch et al. (2004) and decompose the study area into 224 mesh slices (224 processors) for parallel computing based upon the message-passing interface (MPI) (Gropp et al., 1996). The average distance between the GaussLobatto-Legendre integration points at the surface of the model is about $2 \mathrm{~m}$, that is, small enough to resolve the LiDAR DTM data at a maximum frequency of approximately $10 \mathrm{~Hz}$. For the simulation of a hypothetical earthquake occurring in the region, a double-couple point source located at a depth of $4.92 \mathrm{~km}$ and, with a Gaussian source-time function with a half duration of $0.05 \mathrm{sec}$, is located close to the center of the model. The total number of spectral elements and Gauss-Lobatto-Legendre integration points in the model is 3.72 million and 248.07 million, respectively. The timestep is $0.0001 \mathrm{sec}$, and the total duration of the simulation is $5.0 \mathrm{sec}$, that is, 50,000 timesteps. These simulations require 164.5 gigabytes of memory and the average wall-clock time is $23 \mathrm{hr}$.

\section{Snapshots of the Wave Field}

Snapshots of the north $(\mathrm{N})$, east $(\mathrm{E})$, and vertical $(\mathrm{Z})$ components of the velocity wave field are displayed in Fig- 
ure 4 . The $P$ wave reaches the surface with relatively weak amplitude at approximately $1.2 \mathrm{sec}$. The undulating topography reflects and scatters the seismic energy, resulting in complex wavefronts in the area. This can be clearly seen at $2.15 \mathrm{sec}$, when a large amount of reflected and scattered energy emerging after the $S$ wave reaches the surface, especially along mountain ridges and valleys. The wavefront of the $S$ wave is also significantly distorted when it passes through Shamao Mountain. After $2.25 \mathrm{sec}$, the main bodywave phases have propagated through most of the study area; however, the region continues to shake for several seconds. Note that Shamao Mountain traps seismic energy and continues to reverberate. For a very short period of time, Shamao Mountain is concentrically shaken, especially on the two horizontal components. This indicates that the whole mountain reverberates with a period of about $0.1 \mathrm{sec}$.

Next, we consider three models to investigate the influence of topography on ground motion for different surface resolutions. The three models are (a) a flat surface with a 3D background wave-speed model, (b) $40 \mathrm{~m}$ DEM at the sur- (a) East component
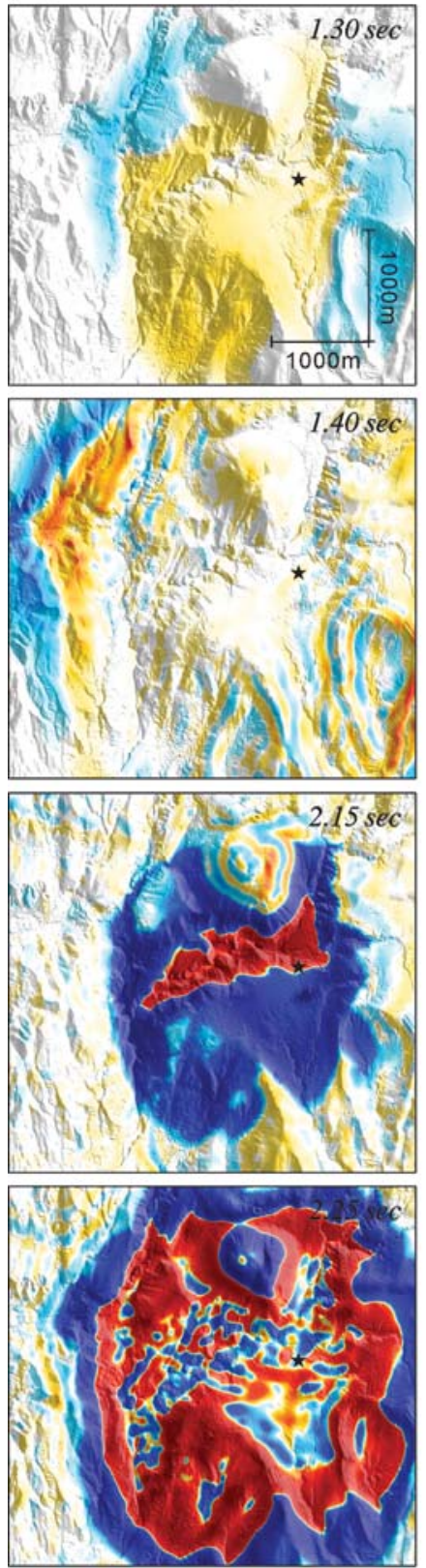

(b) North component
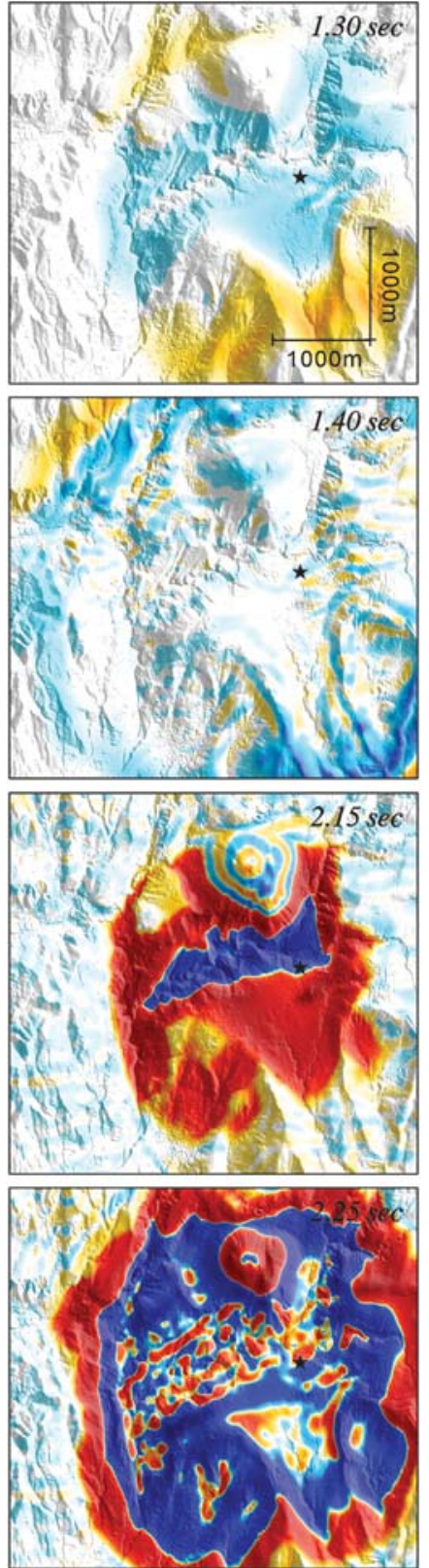

(c) Vertical component
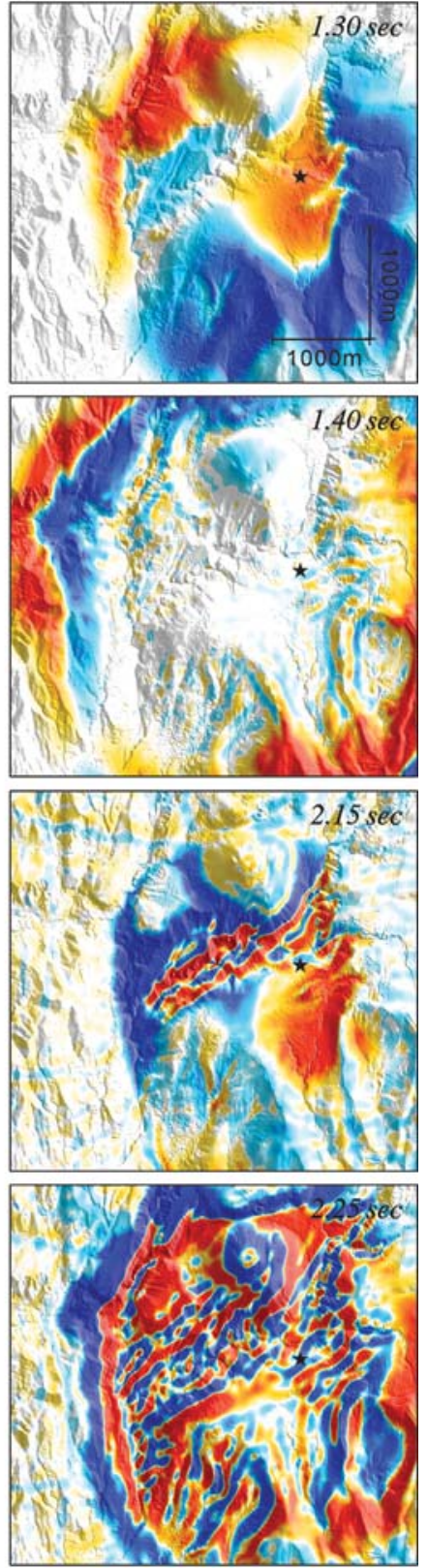

Figure 4 Snapshots of the three components of the wave field for a simulation of a hypothetical double-couple source located beneath the study area at a depth of $4.92 \mathrm{~km}$ : (a) east component, (b) north component, and (c) vertical component. Warm colors indicate positive values and cold colors indicate negative values. The wavefronts are significantly distorted. They are reflected and scattered by steep topography, especially the horizontal components. 
face with a 3D wave-speed model, and (c) LiDAR DTM at the surface with a 3D background wave-speed model. Snapshots of the north component of the seismic-wave field are shown in Figure 5. It is clear that the flat surface model does not exhibit any distorted seismic wavefronts when the waves pass through. The $40 \mathrm{~m}$ DEM and the LiDAR DTM models both exhibit more complex wave propagation patterns that involve complicated reflections and scattering from the mountains. However, the simulation based upon the LiDAR DTM model contains more details for these complicated wavefronts; the amplitudes of the reflected and scattered waves are larger than for the $40 \mathrm{~m}$ DEM model.

\section{Peak Ground Shaking Distribution}

We compare peak ground acceleration (PGA) maps with corresponding surface topographic features present in the LiDAR DTM and $40 \mathrm{~m}$ DEM in Figures 6a,b, respectively. The PGA values are calculated from the norm of the three components of the acceleration vector. Again, the result (a) Half space
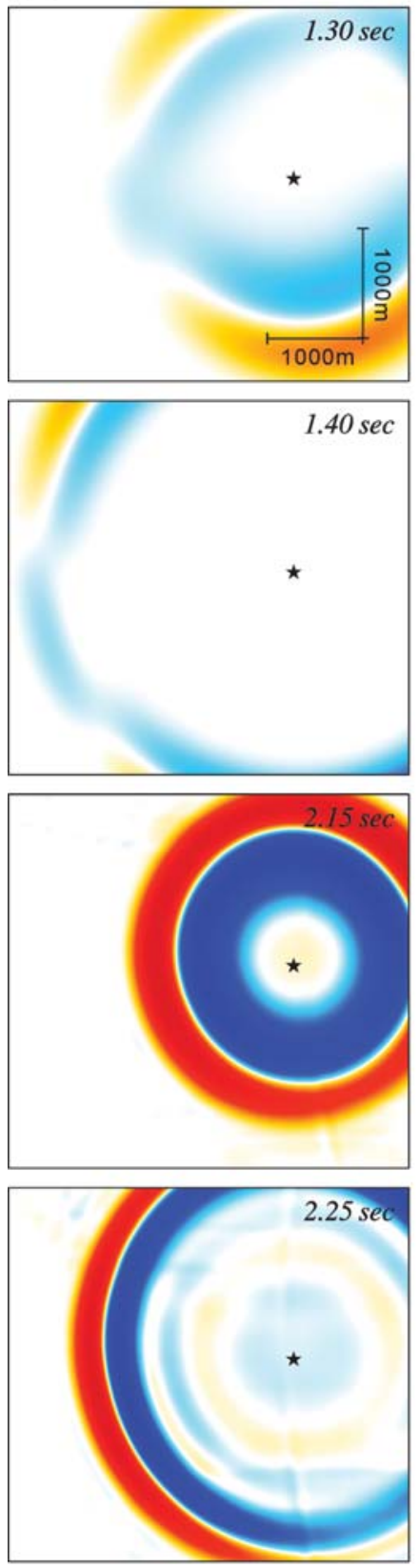

(b) $40 \mathrm{~m} \mathrm{DEM}$
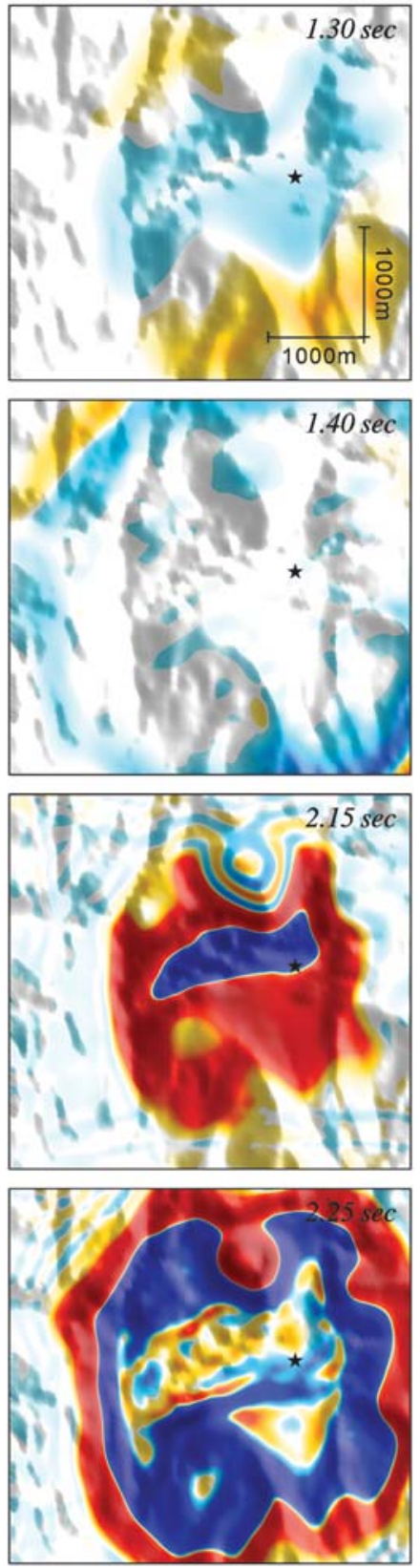

(c) LiDAR DTM
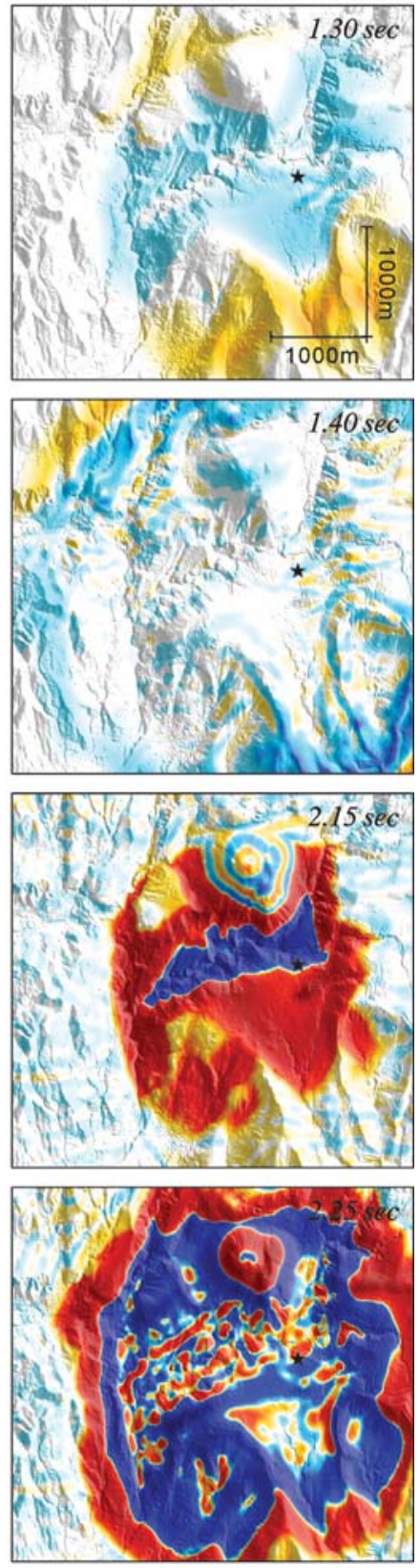

Figure 5. Snapshots of the north component of the wave field for three models: (a) 3D wave-speed model with a flat ground surface, (b) 3D wave-speed model with $40 \mathrm{~m}$ DEM topography, and (c) 3D wave-speed model incorporating LiDAR DTM. Warm colors indicate positive values and cold colors indicate negative values. The epicenter of the double-couple source is represented by a star. 

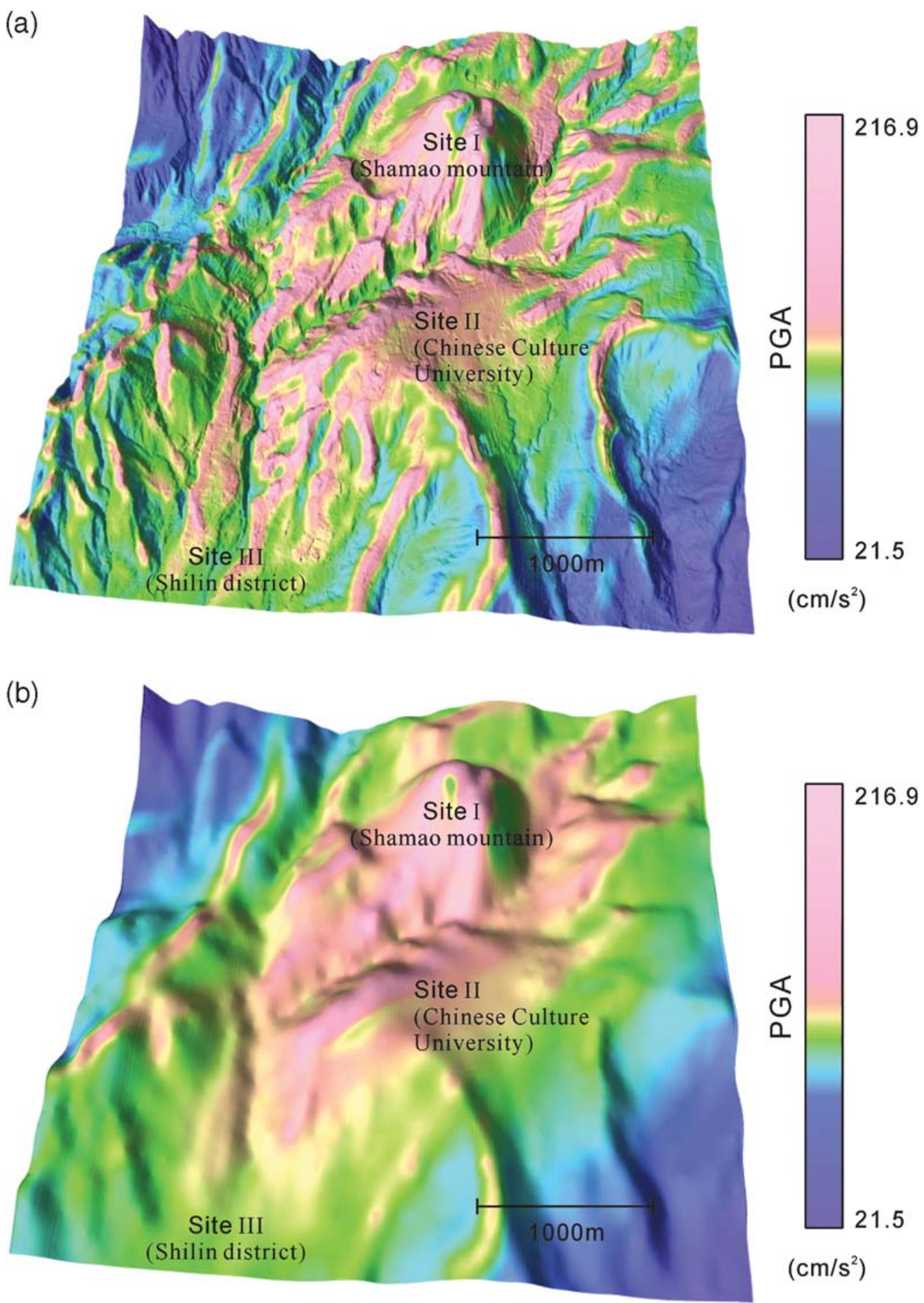

Figure 6. Peak ground acceleration (PGA) comparison for (a) the LiDAR DTM and (b) the $40 \mathrm{~m}$ DEM. The simulated PGA values are computed using the norm of the acceleration vector and are drawn using the color scale shown in the right-hand side of the figure. The topographic data incorporated within each simulation are shown as the background. Three sites are labeled: Shamao Mountain (site I), the Chinese Culture University located on a hilltop (site II), and the Shilin district at the foot of the mountains (site III).

based upon the LiDAR model shows higher resolution and stronger peak ground acceleration patterns. Because we used a double-couple source, the radiation changes with azimuth. In this case, both source radiation and surface topography dominate the PGA distribution at the ground surface. In gen- eral, large PGA values are located close to the center of the study area, with a northeast-southwest expression. However, a complex PGA distribution is observed with relatively large PGA values near the top of mountain ridges and weaker PGA values in valleys and brooks. Large PGA values also occur in 
lower elevation areas such as Shilin (site III in Fig. 6), which is located at the foot of the mountains. Another interesting PGA area is found at Shamao Mountain (site I in Fig. 6), where PGA values show a radiation pattern centered on the top of the mountain. The campus of the Chinese Culture University (site II in Fig. 6) also shows large PGA values because the site is close to the epicenter.

\section{Waveform Comparison}

We investigate the effects of topography on simulated ground motion at two resolutions: one based upon the $40 \mathrm{~m}$ DEM and another based upon the $2 \mathrm{~m}$ LiDAR DTM. The synthetic waveforms obtained from the model with a flat surface are also used for comparison. Sample synthetic waveforms for the vertical component of velocity at five different sites are shown in Figure 7 . The first arrivals ( $P$ wave) are almost identical in all three models, except that the travel-time determined from the flat surface model is slightly different from the other two models, while the amplitudes of the later phases noticeably differ. At the top of Shamao Mountain (site 1), several large-amplitude later phases arrive after the $S$ wave. These phases are related to reverberations occurring at the top of the mountain, as shown in the previous snapshot analysis. At a hilltop (site 2), there are several strong later phases whose amplitudes are much larger than the $S$ wave; they are not observed in the flat surface synthetic seismograms. The hillside (site 3 ) shows a similar result. For sites 4 and 5, located at the foot of the mountains and in a valley, respectively, the $S$ wave and later phases dominate the overall waveforms. The strong later arrivals are complex at site 4 , which is probably due to the constructive and destructive interference between the major waves. It is worth noting that at site 5, located in a valley, the peak amplitude in the flat model is larger than in the two models with surface topography. In general, the later phases exhibit significantly larger amplitudes in mountainous areas in simulations based upon the LiDAR DTM model.
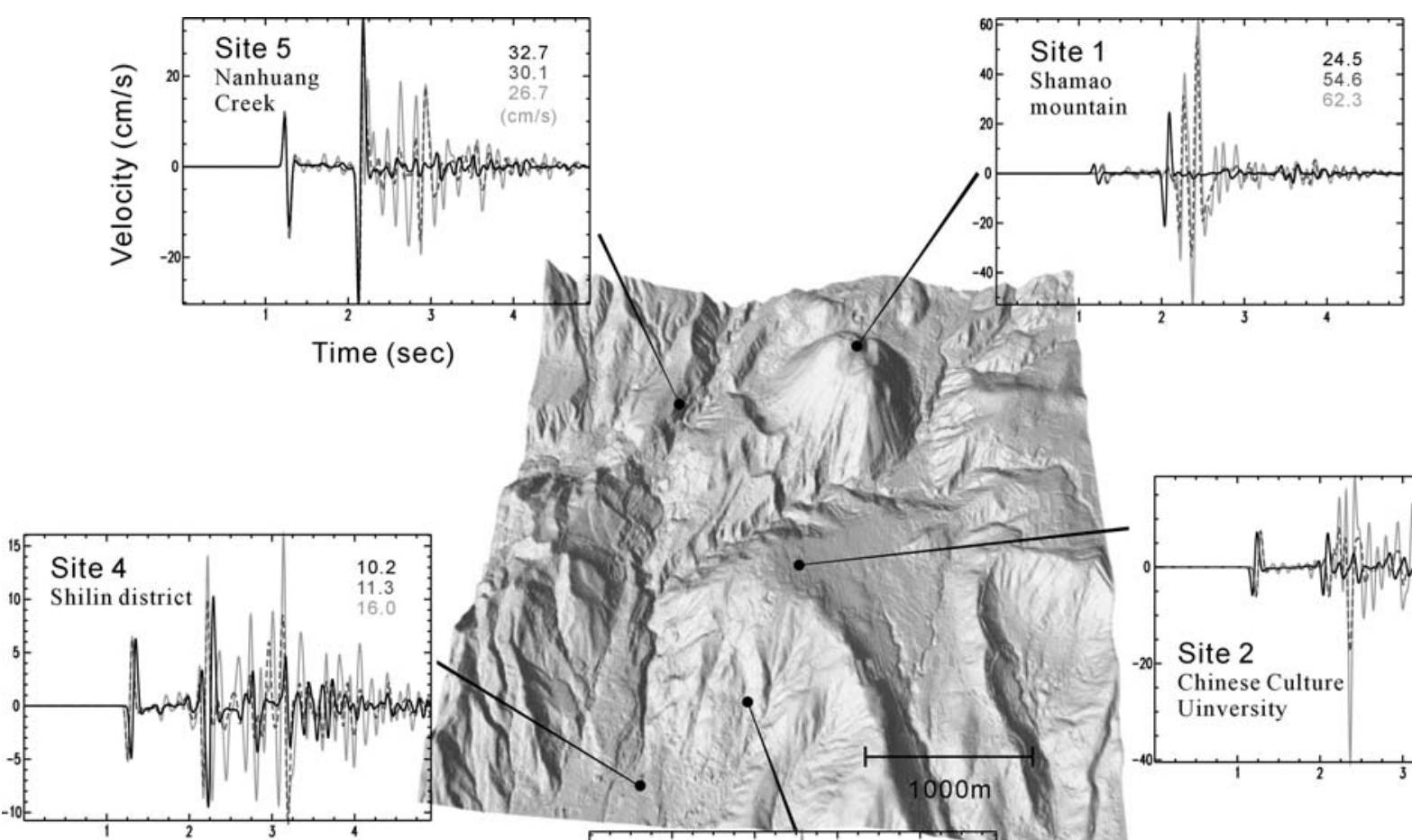


\section{Discussion}

From our scenario earthquake simulation results, we determine the main characteristics of the influence of topography on ground motion: more complex wave propagation and a more complex PGA distribution pattern. To further investigate the effects of realistic topography, we subtract the PGA value for the model without topography from the value for the model with topography, dividing the result by the PGA value for the model without topography, and multiplying it by 100 to obtain a percentage. The value obtained is called the PGA amplification factor. Based upon this approach, we can approximately eliminate the influence of source effects (including both source radiation and geometrical spreading) and evaluate the relative changes between the models with and without topography.

\section{Relative Change in PGA}

Figure 8a illustrates the distribution of the relative changes in PGA values. In general, surface topography increases PGA values at mountain tops and ridges, whereas valleys usually have a reduced PGA, that is, a negative PGA amplification factor. But this is not always true: for example, the PGA value decreases rapidly just beneath the tops of mountain ridges. Increased PGA values were also found in some parts of valleys where brooks eroded the ground surface, resulting in steep topography. PGA amplification values generally vary on the order of $\pm 50 \%$, and the difference in PGA amplification between a valley and a ridge can be as high as a factor of 2 . The peak amplification values are a bit larger than the results obtained by Lee, Chen, Liu, et al. (2008), who considered a coarser topography model taken from the $40 \mathrm{~m}$ DEM. Note that PGA amplification at the campus of the Chinese Culture University (area II in Fig. 8a) is not high, even though the campus is situated on a hill.

\section{Cumulative Kinetic Energy}

Surface topography not only changes peak values of ground shaking but also increases the duration of shaking because of scattered and reflected waves. To measure the relative change in ground-motion duration, we consider another parameter named $E_{k}$, or cumulative kinetic energy. This parameter is obtained by integrating the square of the velocity amplitude of all three components over time and multiplying by half of the density. $E_{k}$ reflects the amplitude and duration of total motion, which may be more closely related to potential damage from shaking (Olsen et al., 1995; Lee, Chen, and Huang, 2008). In our simulations, topography increases $E_{k}$ almost everywhere (Fig. 8b). Again, mountain tops and ridges increase $E_{k}$ dramatically, but valleys and the flat parts on hills also show an increased $E_{k}$. The average $E_{k}$ value in mountainous areas is about one fold that of the value obtained when we use a flat surface; in some areas, it increases by over $200 \%$. There are only a few areas that show decreased $E_{k}$. This is attributed to the fact that when the ground surface is flat, all of the upgoing waves are reflected back into the Earth. However, when topography is incorporated, waves are still reflected but some of the energy horizontally propagates and continues to interact with the surface topography. This is the main reason why $E_{k}$ increases in the presence of topography. This phenomenon can also be clearly observed in the surface wave field snapshot analysis shown in Figure 5a,c.

\section{Realistic Topography Effects on Ground Motion}

We further investigate topographic effects on seismic ground motion due to variations in (a) source frequency, (b) source depth, (c) wave-field type, and (d) the subsurface model by analyzing the PGA amplification factor along profile A-A' in Figure 8a.

Three different dominant source frequencies, 1, 5, and $10 \mathrm{~Hz}$, are considered in test (a), which is summarized in Figure 9a. A higher source frequency results in more smallscale perturbations and larger peak values along the profile. To the contrary, lower source frequencies result in a smooth distribution of PGA amplification. This result is due to the fact that high-frequency waves interact with small-scale topography, which further amplifies the peak values. During past earthquakes (such as the 1995 Kobe earthquake and the 1999 Chi-Chi earthquake), localized strong shaking spots have been reported, where some buildings experienced serious damage while adjacent ones did not (Ouchi and Huang, 1998; Ouchi et al., 2001). This phenomenon could be caused by the interaction between small-scale topography and highfrequency surface waves, which can produce unusually large shaking.

In the analysis of source depth in test (b), we consider three different hypocenter depths: 2,5 , and $8 \mathrm{~km}$. The results in Figure 9b show similar PGA amplification patterns in each of the three cases. The amplification for a $2 \mathrm{~km}$ source depth exhibits slight differences relative to the deeper sources, but the overall pattern is the same. These results indicate that source depth does not have a significant influence on the PGA amplification in our study area.

A double-couple source, explosive source, and a vertically incident plane $P$ wave are compared in test (c). The PGA amplification values show different distributions for different source types, especially at hilltops (area 3). In general, the PGA amplification factor for a double-couple source has larger localized peak values along the profile. Because of the lack of $S$ waves in the case of an explosive source and for a vertically incident plane $P$ wave, these two scenarios do not produce ground motions that are relevant for realistic estimates of surface topography effects.

The accuracy of the simulation strongly depends on both the resolution of topography and the 3D wave-speed model. However, it is not easy to obtain a detailed subsurface 

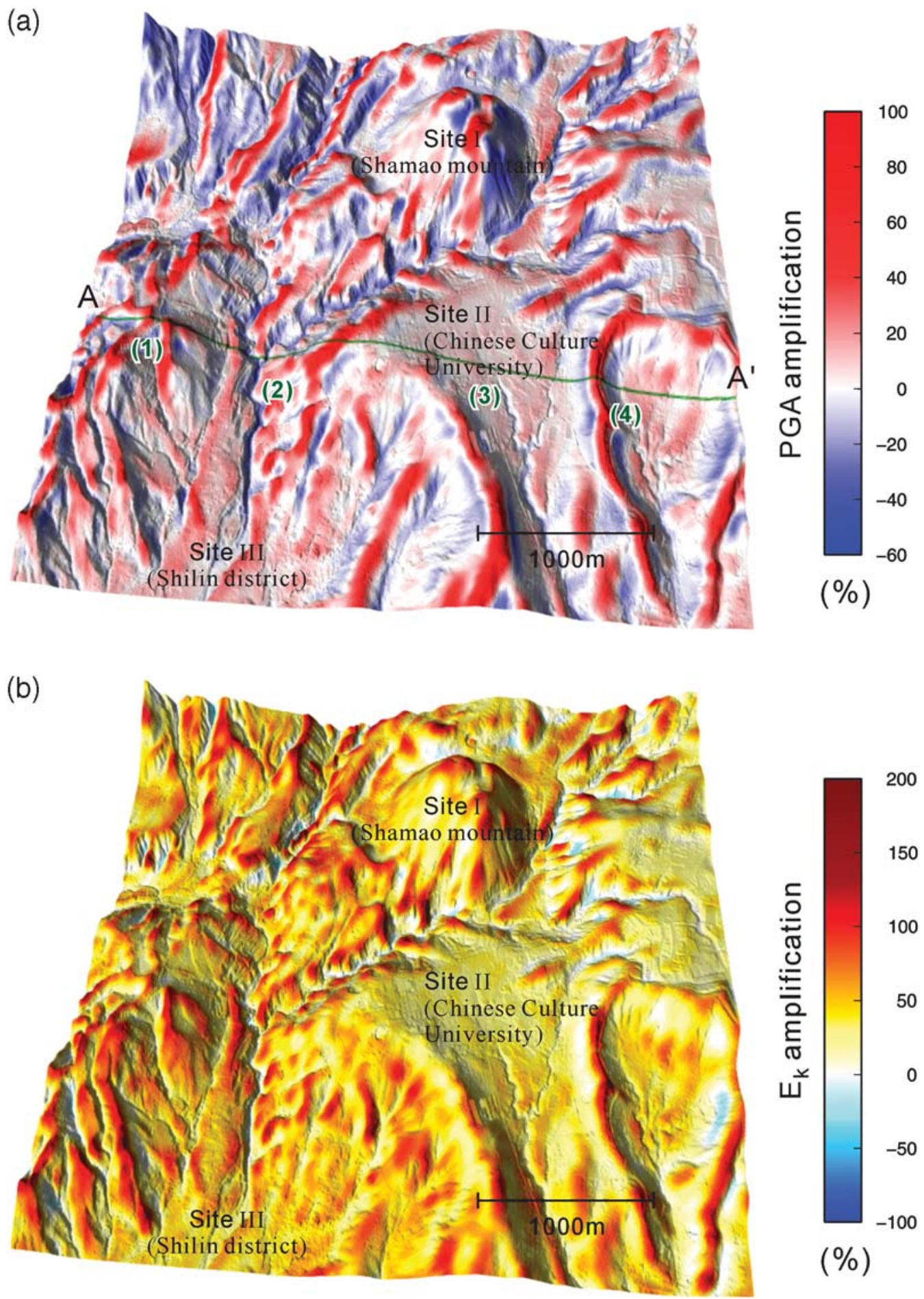

Figure 8. Amplification due to surface topography: (a) relative change in peak ground acceleration and (b) relative change in cumulative kinetic energy $E_{k}$. The amplification factor is obtained by subtracting the peak value for the model without topography from the value for the model with topography, dividing the result by the peak value for the model without topography, and multiplying the result by 100 to obtain a percentage. The amplification values are labeled according to the color legend in the right-hand side of the figure. The same three sites as in Figure 6 are indicated. Profile A-A' and four specific locations situated along the profile are highlighted in Figure 8a. 

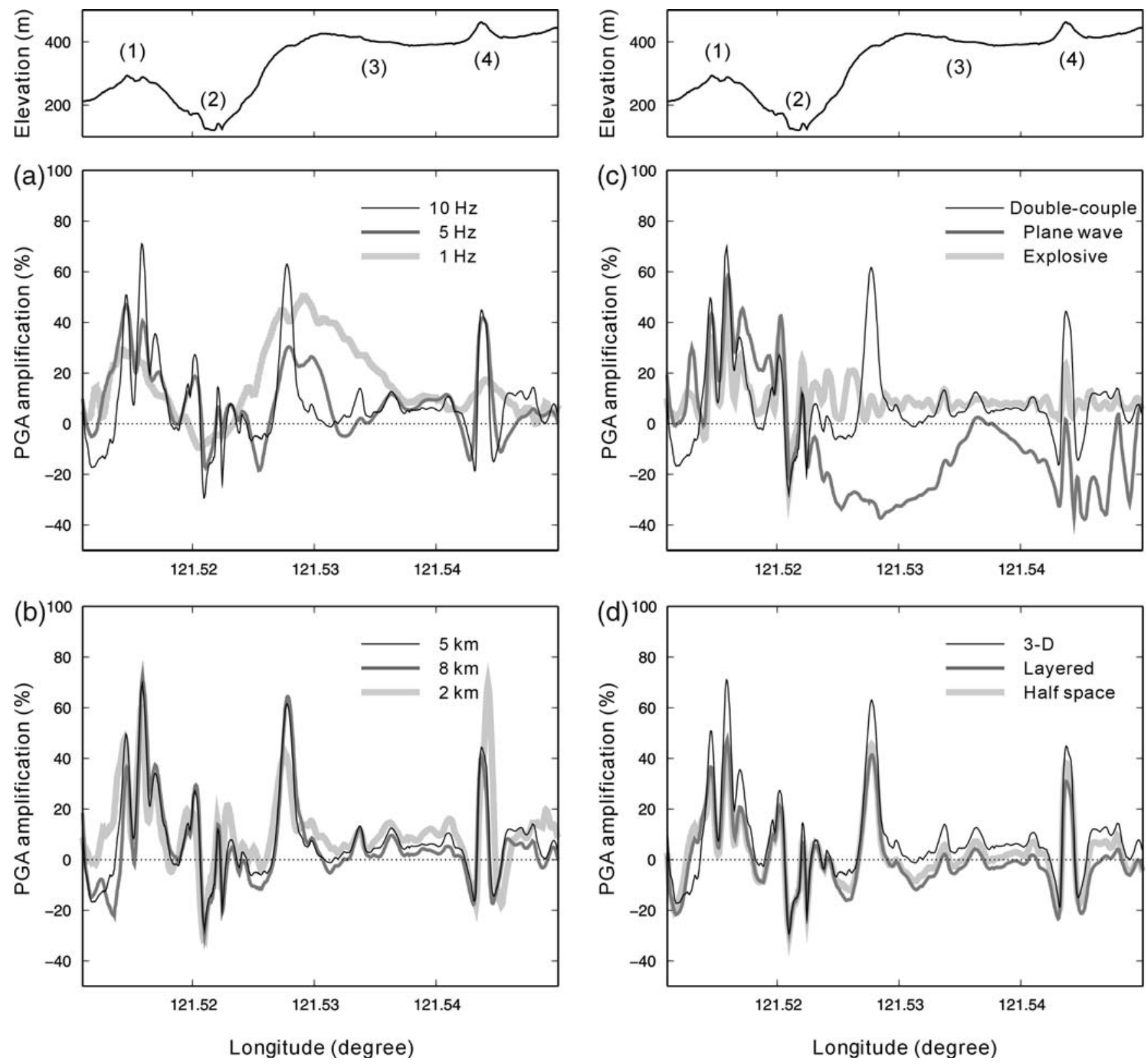

Figure 9. Effects due to variations in (a) source frequency, (b) source depth, (c) input wave-field type, and (d) subsurface structure by analyzing the relative change in PGA along profile A-A' in Figure 8a. The upper panels show the related cross section of the LiDAR DTM. Four specific locations are labeled by numbers along the profile.

wave-speed model in mountainous areas. Here we examine the influence of subsurface structure by considering different wave-speed models: a homogeneous model $\left(V_{P}=\right.$ $3.5 \mathrm{~km} / \mathrm{sec}, V_{S}=2.0 \mathrm{~km} / \mathrm{sec}$ ), a layered model (from the free surface to about $2000 \mathrm{~m}$ depth: $V_{P}=4.0 \mathrm{~km} / \mathrm{sec}$, $V_{S}=2.3 \mathrm{~km} / \mathrm{sec}$; below $2000 \mathrm{~m}: V_{P}=4.5 \mathrm{~km} / \mathrm{sec}$ and $V_{S}=2.5 \mathrm{~km} / \mathrm{sec}$ ) as well as a 3D model. For the 3D model (Fig. 9d), the PGA amplification exhibits a complex distribution, as discussed in the previous sections. The layered and homogeneous models show similar patterns but have on average slightly lower amplification values along the profile. However, in general, the amplification patterns in all three subsurface models are comparable.
The quality factor $Q$ is an important parameter in modeling of seismic-wave propagation. Because of a lack of information regarding attenuation in the study area, we use a shear quality factor, $Q_{\mu}$, of 500 in the bedrock; we do not incorporate bulk attenuation in our analysis. To investigate the effects of larger attenuation near the ground surface, we assume a $Q_{\mu}$ of 90 for the shallow part from the ground surface to about $900 \mathrm{~m}$ depth. Examples of synthetic seismograms recorded on Shamao Mountain determined from models with and without higher attenuation are shown in Figure 10. The results indicate that synthetic waveforms are not very sensitive to the presence of a low shear $Q$ zone underneath the ground surface. 


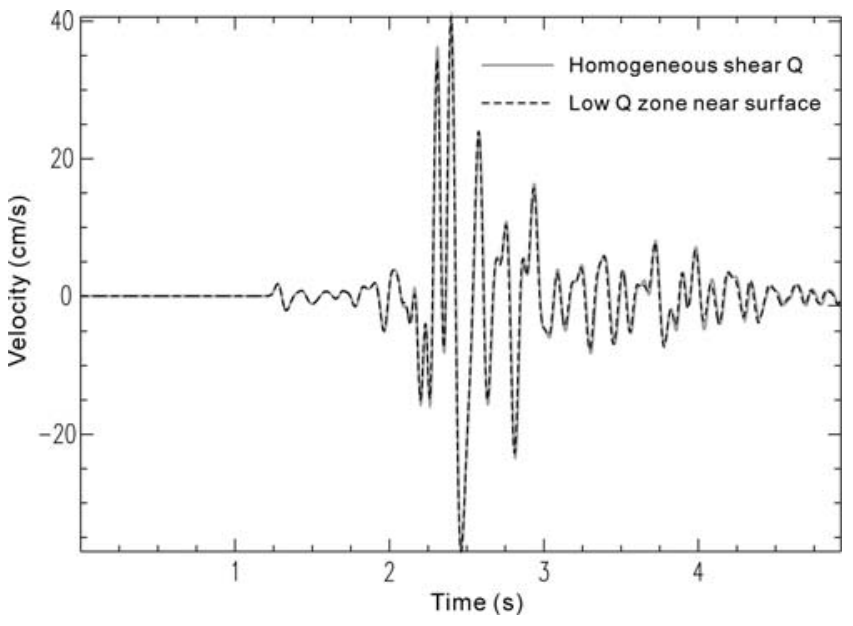

Figure 10. Effect of the shear quality factor $Q_{\mu}$ on simulations: (a) homogeneous $Q_{\mu}=500$ in the whole study area and (b) a higher attenuation zone with $Q_{\mu}$ of 90 from the ground surface to $900 \mathrm{~m}$ depth. We show examples of vertical component velocity waveforms recorded on Shamao Mountain. The solid line corresponds to the result for a homogeneous $Q$ and the dashed line corresponds to the result with a higher attenuation zone. Both waveforms are filtered between 0.1 and $10 \mathrm{~Hz}$.

\section{Conclusions}

We combined LiDAR DTM data and an improved spectral-element mesh implementation to accommodate high-resolution topography in the Yangminshan region in northern Taipei. The average distance between points at the top of the SEM mesh was approximately $2 \mathrm{~m}$, which enabled us to include realistic topography from LiDAR data and was sufficient to calculate the response of seismic waves up to a maximum frequency of approximately $10 \mathrm{~Hz}$. The numerical results showed that topography increases PGA values at mountain tops and ridges, whereas valleys usually have a reduced PGA. In some locations, the PGA value rapidly decreases just beneath the tops of mountain ridges. Increased PGA values are also found in parts of valleys where brooks have eroded the ground surface, resulting in steep topography. Topographic effects also strongly depend on the source frequency content. High-frequency waves can interact with small-scale topography and further amplify peak values. In general, our results demonstrate that topography can change PGA values by $\pm 50 \%$ in mountainous areas, and the relative change in PGA between a valley and a ridge can be as high as a factor of 2 compared to a flat surface response. Furthermore, the mountains can produce complex patterns of reflected and scattered waves, which significantly increase the duration of shaking. The average cumulative kinetic energy $E_{k}$ value increases about onefold in the mountains, and in some areas, it exhibits a more than $200 \%$ increase over the values obtained for a flat surface. The results demonstrate that high-resolution realistic surface topography needs to be taken into account for seismic hazard analysis in densely populated mountain areas.

\section{Data and Resources}

We used the SPECFEM3D software package (available at www.geodynamics.org) developed by Komatitsch et al. (2004) in this study. The LiDAR-derived DTM was reprocessed from the raw LiDAR data funded by the Central Geological Survey, Taiwan, to Y. C. Chan. These data cannot be released to the public.

\section{Acknowledgments}

This research was supported by the Taiwan Earthquake Research Center (TEC) funded through the National Science Council (NSC) with Grant Number NSC95-2745-M-001-004 and the U.S. National Science Foundation under Grant Number 0711177. The simulations are carried out on Caltech's CITerra Dell cluster. The TEC contribution number for this article is 00049. This is Contribution Number 8993 of the Division of Geological and Planetary Sciences, California Institute of Technology.

\section{References}

Boore, D. M. (1972). A note on the effect of simple topography on seismic SH waves, Bull. Seismol. Soc. Am. 62, 275-284.

Boore, D. M., S. C. Harmsen, and S. T Harding (1981). Wave scattering from a steep change in surface topography, Bull. Seismol. Soc. Am. 71, 117-125.

Chaljub, E., Y. Capdeville, and J. P. Vilotte (2003). Solving elastodynamics in a fluid-solid heterogeneous sphere: a parallel spectral element approximation on non-conforming grids, J. Comput. Phys. 187, no. 2, 457-491.

Chan, Y. C., Y. G. Chen, T. Y. Shih, and C. Huang (2007). Characterizing the Hsincheng active fault in northern Taiwan using airborne LiDAR data: detailed geomorphic features and their structural implications, J. Asian Earth Sci. 31, 303-316, doi 10.1016/j.jseaes 2006.07.029.

Chan, Y. C., J. Lee, R. Chen, J. Liu, W. Hsu, J. Hu, W. Chen, Y. Chen, D. Cheng, and S. Tsao (2005). Airborne laser swath mapping of the metropolitan Taipei area (Abstract T32B-06), Eos Trans. AGU 86, no. 52 (Fall Meet. Suppl.), T32B-06.

Chiu, H. C., and H. C. Huang (1992). Effects of the canyon topography on ground motion at the Feitsui damsite, Bull. Seismol. Soc. Am. 82, 1646-1660.

Geli, L., P. Y. Bard, and B. Jullien (1988). The effect of topography on earthquake ground motion: a review and new results, Bull. Seismol. Soc. Am. 78, 42-63.

Gropp, W., E. Lusk, N. Doss, and A. Skjellum (1996). A high-performance, portable implementation of the MPI message passing interface standard, Parallel Comput. 22, 789-828.

Hartzell, S. H., D. L. Carver, and K. W. King (1994). Initial investigation of site and topographic effects at Robinwood Ridge, California, Bull. Seismol. Soc. Am. 84, 1336-1349.

Huang, B. S. (2000). Two-dimensional reconstruction of the surface ground motion of an earthquake: the September 21, 1999, Chi-Chi, Taiwan earthquake, Geophys. Res. Lett. 27, 3025-3028.

Kim, K. H., J. M. Chiu, J. Pujol, K. C. Chen, B. S. Huang, Y. H. Yeh, and P. Shen (2005). Three-dimensional $V_{P}$ and $V_{S}$ structural models associated with the active subduction and collision tectonics in the Taiwan region, Geophys. J. Int. 162, 204-220.

Komatitsch, D., and J. Tromp (1999). Introduction to the spectral-element method for 3-D seismic wave propagation, Geophys. J. Int. 139, 806-822.

Komatitsch, D., and J. P. Vilotte (1998). The spectral-element method: an efficient tool to simulate the seismic response of 2D and 3D geological structures, Bull. Seismol. Soc. Am. 88, no. 2, 368-392. 
Komatitsch, D., J. Ritsema, and J. Tromp (2002). The spectral-element method, Beowulf computing and global seismology, Science 298, $1737-1742$.

Komatitsch, D., S. Tsuboi, and J. Tromp (2005). The spectral-element method in seismology, in Seismic Earth: Array Analysis of Broadband Seismograms, Levander, A., and G. Nolet (Editors), Geophysical Monograph Series Vol. 157, American Geophysical Union, Washington, D. C., 205-227.

Komatitsch, D., Q. Liu, J. Tromp, P. Süss, C. Stidham, and J. H. Shaw (2004). Simulations of ground motion in the Los Angeles basin based upon the spectral-element method, Bull. Seismol. Soc. Am. 94, 187-206.

Lee, S. J., H. W. Chen, and B. S. Huang (2008). Simulations of strong ground motion and 3D amplification effect in the Taipei Basin by using a composite grid finite-difference method, Bull. Seismol. Soc. Am. 98, 1229-1242, doi 10.1785/0120060098.

Lee, S. J., H. W. Chen, Q. Liu, D. Komatitsch, B. S. Huang, and J. Tromp (2008). Three-dimensional simulations of seismic-wave propagation in the Taipei basin with realistic topography based upon the spectralelement method, Bull. Seismol. Soc. Am. 98, 253-264, doi 10.1785/ 0120070033.

Lin, C. H., K. I. Konstantinou, W. T. Liang, H. C. Pu, Y. M. Lin, S. H. You, and Y. P. Huang (2005). Preliminary analysis of volcanoseismic signals recorded at the Tatun Volcano Group, northern Taiwan, Geophys. Res. Lett. 32, no. 10, L10313, doi 10.1029/2005GL022861.

Lin, K. I., C. H. Konstantinou, H. C. Pu, C.C. Hsu, Y. M. Lin, S. H. You, and Y. P. Huang (2005). Preliminary results of seismic monitoring at Tatun volcanic area of northern Taiwan, TAO 6, no. 3, 563-577.

Ma, S., R. J. Archuleta, and M. T. Page (2007). Effects of large-scale surface topography on ground motion, as demonstrated by a study of the San Gabriel Mountains, Los Angeles, California, Bull. Seismol. Soc. Am. 97, 2066-2079, doi 10.1785/0120070040.

Olsen, K. B., R. J. Archuleta, and J. R. Matarese (1995). Three-dimensional simulation of a magnitude 7.75 earthquake on the San Andreas fault, Science 270, 1628-1632.

Ouchi, T., and Q. Huang (1998). Mechanism of strong motions: generation of localized motions, Rep. Res. Cent. Urban Saf. Secur. 2, 123-128 (in Japanese).
Ouchi, T., A. Lin, and T. Maruyama (2001). The 1999 Chi-Chi (Taiwan) earthquake: earthquake fault and strong motions, Bull. Seismol. Soc. Am. 91, 966-976.

Patera, A. T. (1984). A spectral-element method for fluid dynamics: laminar flow in a channel expansion, J. Comput. Phys. 54, 468-488.

Scott, A. A., N. Sitar, J. Lysmer, and N. Deng (1997). Topographic effects on the seismic response of steep slopes, Bull. Seismol. Soc. Am. 87, 701-709.

Wehr, A., and U. Lohr (1999). Airborne laser scanning — an introduction and overview, ISPRS J. Photogramm. Remote Sens. 54, 68-82.

Institute of Earth Sciences

Academia Sinica

Nankang, Taipei 115

Taiwan, Republic of China

sjlee@earth.sinica.edu.tw

yuchang@earth.sinica.edu.tw

hwbs@earth.sinica.edu.tw

(S.-J.L., Y.-C.C., B.-S.H.)

Department of Geophysical Modeling and Imaging in Geosciences University of Pau

CNRS UMR

5212 INRIA Magique 3D

64013 Pau Cedex, France

dimitri.komatitsch@univ-pau.fr

(D.K.)

Seismological Laboratory

California Institute of Technology

Pasadena, California 91125

jtromp@gps.caltech.edu

jtromp@Princeton.edu

(J.T.) 